\title{
SOLAR CORONAL MASS EJECTIONS
}

\author{
A. J. Hundhausen, D. G. Sime and B. C. Low \\ High Altitude Observatory \\ National Center for Atmospheric Research \\ Boulder, Colorado, USA
}

\begin{abstract}
In addition to the more or less steady solar wind, the Sun also ejects mass in highly time dependent events taking place in the corona once every few days at solar activity minimum and as often as three times a day at solar activity maximum (Hundhausen 1988, Low 1986). These events involve large scale reconfiguration of the corona with an expulsion of some $10^{15} \mathrm{~g}$ of ionized material into interplanetary space. The High Altitude Observatory (HAO) operates a groundbased internally occulted coronagraph at Mauna Loa, Hawaii, with a field of view of the corona from 1.2 to $2.2 \mathrm{R}_{\odot}$ in heliocentric distance, registering polarization brightness. A second instrument at the same site in Hawaii observes the solar limb in $H_{\alpha}$ emission to detect chromospheric material from the limb out to $1.5 \mathrm{R}_{\odot}$. HAO also operates an externally occulted coronagraph/polarimeter onboard the NASA Solar Maximum Mission Satellite (SMM) launched in 1980, capitalizing on the advantage of space with a field of view from 1.5 to $6 \mathrm{R}_{\odot}$ to cover the fainter outer corona. Coronal mass ejections involve magnetic field reconfiguration from high in the corona down to the base lying below $1.1 R_{\odot}$. Important physical insights can be had when simultaneous observations by HAO's three instruments are put together with a common scale and orientation to reveal a mass ejection in the full extent of the solar atmosphere from the limb outward. Combined observations of two mass ejections are presented, one associated with an eruptive prominence and the the other associated with a flare. The significance of these two events is that in both cases, the mass ejection was in fully developed motion and had traveled high into the corona well before the onset of the associated prominence or flare eruption, pointing to an instability in the large scale coronal magnetic field as the underlying cause of the global reconfiguration.
\end{abstract}

The National Center for Atmospheric Research is sponsored by the US National Science Foundation and this work is supported by NASA under grant Nos. 555989 and 555989A.

\section{References}

Hundhausen, A. J. 1988, The origin and propagation of coronal mass ejections, in Proc. of Sixth International Solar Wind Conference, edited by V. Pizzo, T. Holzer and D. Sime (NCAR Publ. No. TN-306).

Low, B. C. 1986, Coronal mass ejections, in Highlights of Astronomy, edited by J. P. Swings (Reidel). 